\title{
A songbird animal model for dissecting the genetic bases of autism spectrum disorder
}

\author{
S. Carmen Panaitof \\ Department of Biology, University of Nebraska at Kearney, Kearney, NE, USA \\ E-mail: carmen.panaitof@gmail.com
}

\begin{abstract}
The neural and genetic bases of human language development and associated neurodevelopmental disorders, including autism spectrum disorder (ASD), in which language impairment represents a core deficit, are poorly understood. Given that no single animal model can fully capture the behavioral and genetic complexity of ASD, work in songbird, an experimentally tractable animal model of vocal learning, can complement the valuable tool of rodent genetic models and contribute important insights to our understanding of the communication deficits observed in ASD. Like humans, but unlike traditional laboratory animals such as rodents or non-human primates, songbirds exhibit the capacity of vocal learning, a key subcomponent of language. Human speech and birdsong reveal important parallels, highlighting similar developmental critical periods, a homologous cortico-basal ganglia-thalamic circuitry, and a critical role for social influences in the learning of vocalizations. Here I highlight recent advances in using the songbird model to probe the cellular and molecular mechanisms underlying the formation and function of neural circuitry for birdsong and, by analogy, human language, with the ultimate goal of identifying any shared or human unique biological pathways underscoring language development and its disruption in ASD.
\end{abstract}

Keywords: Autism spectrum disorder, animal model, FOXP2, CNTNAP2, song circuitry, vocal learning

\section{Introduction}

Despite decades of research into the neurobiological basis of human language and communication disorders, we have an incomplete understanding of the molecular and cellular underpinnings of socially-learned vocal communication. Thanks to considerable progress in the field of human neurogenetics, it has become possible to map an increasing number of neural disorders onto abnormal genetic variants [1,2]. Of particular relevance to understanding the neurobiology of human speech and language are neurological syndromes with developmental onset in which language is affected, such as autism spectrum disorder (ASD). ASD encompasses a range of neurodevelopmental disorders of varying severity that involve impairments in three core social domains: language development and use, behavioral flexibility, and reciprocal social interaction. Within ASD, autism disorder (the most severe end of the spectrum) and pervasive developmental disorder - not otherwise specified represent the most common clini- cal manifestations of the disease [3]. ASD has a strong genetic basis and robust heritability, with current prevalence estimates indicating that 1 in 166 children are diagnosed with the disorder each year [3]. In addition to the phenotypic variation associated with the degree of severity in the clinical presentation of the disease, a growing list of gene mutations and genetic structural variation linked to autism susceptibility [4,5] demonstrate that ASD is also a genetically heterogeneous disorder. Both rare and common genetic variants have been shown to modulate disease risk and it is currently estimated that no single genetic cause of autism may account for more than 1-2\% of ASD cases [4,5]. However, the increasing realization that single gene lesions can be sufficient in causing ASD suggests that the search for rare mutations associated with ASD will remain a powerful tool in deciphering the etiology of the disease $[4,5]$. Given the behavioral and genetic complexity underlying ASD, the task of selecting appropriate animal models in which to study the disease can be particularly challenging. Appropriate selection of animal 
models of ASD starts from the premise that core autism features such as the deficits of social interaction and impaired communication can be adequately assayed in animals and that these behavioral deficits represent a good approximation of the underlying neurobiological processes disrupted in ASD.

In this review, I outline the significance of a nonhuman vocal learner, the zebra finch songbird species, in exploring the neural and genetic mechanisms subserving the capacity for learned vocal communication that songbirds share with humans but not traditional laboratory animals such as rodents or non-human primates. Given the powerful analogy between birdsong and human speech, studies probing the link between autism-susceptibility and language-related genes, and vocal learning, in the experimentally tractable songbird model, hold tremendous promise in identifying any unique or shared neuromolecular pathways enabling learned vocalization. Uncovering basic biological mechanisms at play during vocal development has in turn implications for designing controlled molecular and cellular studies to shed light onto the biological pathways disrupted in communication disorders like ASD.

\section{Modeling autism features in animals}

\subsection{Genetic models of ASD}

Due to their genetic tractability, rodent animal models of ASD have traditionally proven useful in probing the individual contribution of ASD-related genetic variants to brain development and function. At the same time, gene knock-out and gene knock-in rodent models have provided the opportunity to isolate the effect of specific genetic lesions on the performance of species-typical social behavior, using behavioral testing paradigms relevant for screening for autistic-like deficits. This approach has been successful in the study of both heritable syndromic forms of autism like Fragile X or Rett syndrome and monogenic non-syndromic forms of ASD [8]. In the latter case, for example, mice bearing genetic lesions of SHANK3 ( $\mathrm{SH} 3$ and multiple ankyrin repeat domains 3 ), a gene that encodes a postsynaptic protein present at glutamatergic synapses, exhibit excessive, repetitive grooming and reduced social interactions [6]. SHANK3 mutant animals also show morphological defects of striatal medium spiny neurons, as well as functional impairments at corticostriatal synapses [6]. Mice lacking NEUROLIGIN-4 which encodes a cell adhesion molecule similarly show selective deficits of reciprocal social interactions and impaired communication, quantified as a decrease in ultrasonic vocalizations (USVs) compared to wild-type animals [7].

There are, however, clear limitations in relying exclusively on mouse models in the study of ASD pathogenesis. It is, for instance, unclear to what extent the deficits in communication (e.g. reduced USVs) and reduced social interactions observed in mutant mice can be exclusively circumscribed to an autism-like phenotype, as clearly distinguishable from the behavioral abnormalities assayed in animal models of other neurodevelopmental disorders, for example schizophrenia [8]. At the same time, as illustrated by the studies referred to above, mouse models bearing mutations in autismsusceptibility genes may display some, typically two out of three, but not all, of the core deficits that represent the clinical hallmark of ASD. Finally, given that the disruption in the social function of USVs, which are not known to be learned, may not represent a good approximation of the language regression and linguistic deficits associated with ASD, alternative animal models need to be developed in which to better assay ASDrelated vocal communication deficits.

\subsection{The songbird model of socially-learned vocal communication}

Towards the goal of selecting animal models better suited to assay the functional consequences of genetic lesions in inducing autistic-like vocal communication deficits, songbird researchers can turn to a model system that has important advantages over traditional laboratory animals. For several decades, the zebra finch songbird species has represented the only experimentally tractable animal model for investigating the neural substrate for the formation and function of a specialized, socially-sensitive neural circuitry for vocal learning [9-11]. Vocal learning, the capacity to learn to produce new sounds for communication, is a rare trait across animal groups, with humans and songbirds being the best characterized exemplars. Importantly, traditional laboratory animals such as rodents and non-human primates, while rich with communicative behaviors, are not thought to learn their vocalizations. In songbirds, vocal learning is part of speciescharacteristic behavior and, in some species such as the zebra finch, is sexually dimorphic: during post-hatch brain development, male, but not female, zebra finch, develop a discrete set of interconnected brain nuclei 
dedicated to the learning and production of song [9, 10]. Thus, songbird provides a significant advantage over traditional animal models by enabling the study of an important subcomponent of language, vocal learning, in an experimentally tractable species, with the potential to illuminate how language learning processes and social influences on these developmental processes may become disrupted in ASD.

Song learning and human language acquisition share several key features $[12,13]$. In both songbirds and humans, vocal learning: 1) takes place during critical developmental phases comprised of partially overlapping stages of sensory acquisition and sensorimotor practice; 2) relies upon social interactions; 3) requires specialized brain circuitry dedicated to the development and production of the new sounds. Thus, in addition to its innate vocalizations, during song development a young songbird learns one or more songs from an adult tutor, usually its father. Initially, sensory exposure to a tutor song allows young birds to generate a brain 'template' of the adult tutor song that they attempt to match during the sensorimotor practice stage of song development. Both sensory and sensorimotor phases of song learning are critically dependent on auditory feedback. Like human infants, juvenile songbirds need to hear themselves in order to match their vocal motor output to the tutor template. The initial vocalizations (subsong) of young birds are highly variable and unstable, akin to the babbling of human babies $[12,13]$. Through social interactions with their tutor, juvenile songbirds then refine these vocalizations and their subsong gradually evolves into a plastic song and eventually, as they approach sexual maturity, into crystallized (stable) adult song. Although the unchanging song of adult zebra finches appears to be in marked contrast to the relatively limitless capacity of human language to connect new sounds to meaning, several other songbird species are capable of learning to produce new songs throughout life (e.g. mockingbirds). Further, adult zebra finch song is actively maintained by auditory feedback throughout life and gradually deteriorates in males that are deafened $[11,12]$. The same is true with the human speech in individuals who become deaf as adults [14].

Zebra finches possess cortico-basal ganglia-thalamic circuitry analogous to that of the human, dedicated to the production and modification of fine sequential articulations known as song. In the zebra finch, the brain areas responsible for song have been wellcharacterized, facilitated by their sexual dimorphism, and are collectively referred to as the song circuit [9, 10]. Subregions within the cortical-like pallium, the basal ganglia and thalamus are prominent and interconnected only in male zebra finches. These include the specialized subregions whose neurons are exclusively dedicated to song within each brain region. The song circuit is comprised of two interconnected pathways that underlie the development and production of learned song (Fig. 1). Both pathways stem from the cortical-like (pallial) HVC (acronym used as its proper name), homologous to association cortex in humans. In the first, known as the vocal motor pathway, necessary for song production [15], auditory inputs enter the circuit at HVC, which contains neurons that send axonal projections to another corticallike area known as the robust nucleus of arcopallium (RA). RA, homologous to primary motor cortex, in turn, projects to brainstem motor neurons that control the syrinx or vocal organ and respiratory muscles used in singing [16]. The second pathway, known as the anterior forebrain pathway (AFP), analogous to the cortico-basal ganglia loop of mammals, is required for song learning and modification [17-19]. The AFP begins with area $\mathrm{X}$ (homologous to mammalian basal ganglia), a nucleus in the songbird striatum essential for song learning, which projects to the dorsolateral portion of the medial thalamus (DLM) and then to neurons in the lateral magnocellular nucleus of anterior nidopallium (LMAN), a frontal cortical-like nucleus involved in song modification. The AFP intersects vocal motor pathway via the projection of LMAN to RA and receives input from the latter through the projection of HVC to area X [20-22]. The similarity between mammalian cortico-striato-thalamo-cortical loops and song circuitry extends beyond neuroanatomical connectivity to neuromodulation: area $X$ receives dense dopaminergic input from midbrain nuclei [23], as well as glutamatergic input from cortical-like HVC and LMAN [24]. Similar inputs to the mammalian striatum are critical for motor learning and reward [25,26], suggesting that similar mechanisms might operate in songbird circuitry to reinforce and guide song learning.

\section{Genetic basis of socially-learned vocal communication}

\subsection{Language-related genes and vocal learning}

Thanks to the recent advances in the field of human neurogenetics, a growing list of molecular targets disrupted in human communication disorders $[1,2]$ has supplied important candidate molecules to be tested in 


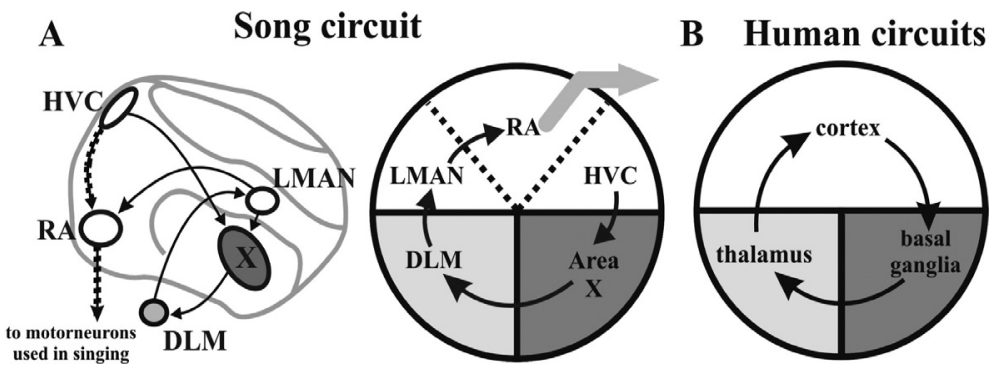

Fig. 1. Schematic comparison of connections within the song circuit and human cortico-basal ganglia-thalamo-cortical circuitry. (A) composite sagittal view of zebra finch telencephalon (left) and schematic view of the song circuit (right). (B) schematic view of the human cortico-basal ganglia-thalamo-cortical circuitry. The cortex and cortical-like pallium are shown in white, basal ganglia in dark gray, and thalamus in light gray. Auditory input (not shown) enters the song circuit at the HVC which relays it to the vocal motor pathway (stippled arrows) and the anterior forebrain pathway (AFP; plain arrows).

the songbird model, with the ultimate goal of revealing basic cellular and molecular underpinnings of human speech and language development, as well as relating these findings to key aspects of pathogenesis in ASD and related language disorders. As a first illustration of this principle, the discovery that a mutation in a single gene, encoding a transcription factor known as FOXP2 (Forkhead box P2), can be directly linked to a severe speech and language disorder [27], has provided a unique opportunity to begin to dissect the genetic substrate of vocal learning using an experimentally tractable model system of socially-learned vocalization. Cross-species comparisons of gene expression between human and non-human vocal learners have indeed revealed that $F O X P 2$ has a remarkably similar pattern of distribution in the developing human and songbird brain, precisely within brain subregions specialized for the learning and production of vocalizations, including both cortical and striatal areas [28].

Importantly, as a transcription factor, FOXP2 is expected to orchestrate the expression of many downstream gene targets, including those implicated in the patterning and functional connectivity of languagerelated areas. This prediction has been recently fulfilled by the discovery and validation of one of the first targets of FOXP2 in human, contactin-associated proteinlike 2 (CNTNAP2) [29-31], an autism susceptibility gene [32-34] that was previously shown to map within a language QTL interval [35]. Importantly, homozygous truncating mutations in CNTNAP2 discovered in a group of Old Order Amish children diagnosed with cortical dysplasia-focal epilepsy (CDFE) syndrome were shown to result in severe deficits that included intractable seizures, language regression and other pervasive ASD-related developmental delays [36]. Subsequently, both common and rare genetic variants of CNTNAP2 have been linked to ASD susceptibility [32-34], including an association between CNTNAP2 variation and a quantitative measure of language delay, age at first word [32]. In addition, CNTNAP2 polymorphisms were significantly associated with nonsense-word repetition, a heritable marker of Specific Language Impairment [31], and copy number variants of CNTNAP2 were shown to segregate with other complex psychiatric disorders such as schizophrenia [37].

The protein encoded by CNTNAP2 is a member of the neurexin superfamily of transmembrane proteins implicated in cell-cell communication in the nervous system. As a neuronal cell adhesion molecule, CNT$N A P 2$ is specifically implicated in neuronal-glia interaction via its well-described role in clustering Shakerlike voltage-gated potassium channels at the juxtaparanodal regions of myelinated axons [38-40]. Although its function in the peripheral nervous system is better understood, very little is known of the role of $C N T$ $N A P 2$ in the central nervous system. Brain tissue resected from CDFE patients revealed abnormal patterns of neuronal migration and altered cytoarchitecture in cortical areas, consistent with a role in cortical histogenesis and laminar organization [36].

During development, CNTNAP2 expression is enriched in human frontal cortex and basal ganglia comprising neural circuitry important for the development of language and other higher cognitive functions [41]. Surprisingly, cross-species comparisons among mammals demonstrated that CNTNAP2 transcript distribution differs between vocal learners and non-vocal learners: its enrichment in language-related cortico-basal ganglia-thalamic circuitry in fetal human brain is in sharp contrast to a broad, non-punctuated distribution in developing mouse and rat brain at comparable embryonic stages [41]. This neuroanatomical specialization in humans is consistent with the genetic links to language, above. Together, these data provides strong 
support for the hypothesis that CNTNAP2 contributes to the regional cortical patterning and functional specialization of speech and language areas in the developing human brain. Importantly, the focal enrichment observed in human cortico-basal ganglia circuitry corresponds to a gestational stage prior to myelination, indicating that additional developmental roles of $C N T$ $N A P 2$ in the central nervous system remain to be elucidated [41]. Consistent with the idea that CNTNAP2 may play additional developmental roles, a recent study by Peñagarikano et al. [42] revealed that the autisticlike behavioral deficits of CNTNAP2 knock-out mice, which included diminished social interactions and reduced USVs, are accompanied by defects in the patterns of neuronal migration of cortical projection neurons, as well as a reduction in the number of GABAergic interneurons.

\subsection{CNTNAP2 expression in songbird}

Towards elucidating the role of CNTNAP2 in the patterning of language-related brain areas and to investigate whether CNTNAP2 is important for learned vocal communication in additional vocal learners, we performed a comprehensive study of in situ hybridization analyses to determine the distribution of CNTNAP2 in songbird brain [43]. Using zebra finch-specific in situ riboprobes we determined that, within the male zebra finch brain, CNTNAP2 transcript distribution punctuates the vocal learning circuitry, consistent with the sexual dimorphism of brain and song learning behavior [43].

In males, but not females, CNTNAP2 transcript shows differential expression within song nuclei compared to surrounding regions which contain similar cell types $[44,45]$. CNTNAP2 is markedly enriched in cortical-like (pallial) song nuclei LMAN, in the anterior forebrain, and RA, in the posterior forebrain. In striatal song nucleus area $\mathrm{X}, C N T N A P 2$ transcript shows striking downregulation compared to surrounding tissue. The diminished expression in area $\mathrm{X}$ is particularly remarkable given that the cell packing density in this song nucleus is higher than in the outlying striatum [10]. Importantly, these changes in transcript levels are not observed in corresponding areas in the female striatum which lacks area X [9]. The differential expression of CNTNAP2 in these brain subregions suggests that both upregulation and downregulation of transcript levels are important for the 'on-line' function of these specialized song-related areas in the adult male songbird.
During post-hatch development, the onset of sexually-dimorphic CNTNAP2 expression takes place at 35 days in the case of song nucleus RA and $\approx 40$ days in the case of song nucleus LMAN [43]. The finding is remarkable given that these time points map onto the beginning of the sensorimotor practice phase of song learning, a stage when young male zebra finches begin to actively practice a tutor's song, suggesting that changes in CNTNAP2 transcript abundance and distribution correlate with the formation and functional activation of these brain structures for song learning and production. Importantly, although in females CNT$N A P 2$ expression in song nuclei LMAN and RA shows a similar enrichment to that observed in males, after 35 days CNTNAP2 transcript levels diminish dramatically in both of these female brain areas. In fact, the differential gene expression pattern observed between male and female brains after 35 days appears to closely track the divergent developmental trajectories of the song nuclei themselves which begin to regress in females precisely at the time ( 35 days) when they become synaptically connected in males [46,47]. Moreover, downregulation of transcript levels in area $\mathrm{X}$ which is observed throughout development and persists into adulthood, conversely indicates that low CNTNAP2 transcript levels in this specialized striatal structure may enable its formation and participation in song learning processes. Together, these results provide support for the hypothesis that CNTNAP2 is important both for the formation and functional specialization of song nuclei for vocal learning during post-hatch development in the male zebra finch. Finally, recent work from Condro et al. [48] consolidated these findings by revealing that the pattern of CNTNAP2 protein distribution in the male songbird brain is remarkably similar to that established for mRNA, with high levels of protein detected in nucleus RA and low levels of protein detected in song nucleus area $\mathrm{X}$ throughout post-hatch development, demonstrating that the active form of the molecule is focally enriched or diminished, correspondingly, at identical key developmental time points during song learning [48].

\subsection{FMRP expression in songbird}

The relevance of the songbird model for probing the genetic substrate of human neural disorders of higher cognition and language has also been recently illustrated by a study by Winograd et al. [49] which focused on the expression of fragile $\mathrm{X}$ mental retardation protein (FMRP) in the zebra finch neural circuitry for song. 
FMRP is encoded by FMRI, the gene mutated in fragile X syndrome (FXS), a common form of X-linked inherited mental retardation [50] that is associated with delays in speech and language development [51,52] and can show co-morbidity with autism [53]. Given the specific speech delays and deficits associated with FXS, the authors hypothesized that disruption of FMRP function is linked to impaired vocal learning and set out to characterize FMRP protein expression during vocal development and performance in songbird. Using a zebra finch specific antibody, the authors showed that FMRP was elevated at 30 days in male song nucleus RA, compared to surrounding telencephalic areas, and protein localization was restricted to neuronal cell types [49]. As in the case of CNTNAP2, the timing for these changes in FMRP abundance corresponds to the beginning of the sensorimotor practice stage of song learning, indicating a role for FMRP in the cellular and synaptic changes unfolding within premotor song nucleus RA at this key time point during song learning. The authors suggest that the pattern of FMRP expression during vocal development in songbird at a stage when song nucleus RA first becomes synaptically connected $[46,47]$ indicates a role for FMRP in song circuit plasticity, with potential implications for understanding the mechanisms at play in the speech and cognitive deficits observed in patients with FXS. Finally, it is important to note that since the speech and language deficits are far better characterized for patients with FXS [51,52] than in the case of patients diagnosed with ASD, ongoing analyses of FMRP and CNTNAP2 function in the songbird model may ultimately lead to complementary approaches in dissecting the molecular pathways underlying impaired vocal communication in the two language disorders.

\subsection{From gene expression studies to in vivo functional investigations}

Taken together, these findings demonstrate that comprehensive studies of gene and protein expression in the songbird model represent a fruitful approach in testing the involvement of language-related molecules in vocal learning. Because the processes of song learning can be easily manipulated in songbird, for example by manipulating the timing or length of exposure to a tutor, it becomes possible to tease out whether changes in gene expression are directly linked to the beginning or end of the critical period for song learning, and whether changes in transcript or protein distribution are responsive to social influences and behavioral state (e.g. singing versus non-singing). In the case of FOXP2, Teramitsu and White [54] demonstrated that transcript levels can indeed be modulated 'on-line', such as when the adult birds sing, raising the possibility of ongoing, post-developmental roles for FOXP2 in humans as well. In the case of CNTNAP2, the role of social influences and behavioral state in modulating transcript and protein levels in songbird neural circuitry for vocal learning, of potential relevance to understanding the social and communication deficits in ASD, remains to be investigated.

At the same time, by providing both key neuroanatomical sites and developmental time points for genetic intervention, determining the patterns of distribution of language-related candidate molecules in the songbird circuitry for learned vocalization can help guide functional in vivo analyses. Given that the formation of neural circuitry for song takes place during post-hatch development, targeted genetic intervention to manipulate gene expression of candidate molecules in select song nuclei is facilitated by the accessibility of these neural structures in the developing songbird brain. Moreover, following targeted genetic manipulation in song-related areas, 'real-time' functional assessment of the behavioral consequences of over- and underexpressing genetic constructs on vocal learning is enabled by reliable quantification of song vocalizations via dedicated sound analysis software.

Thus, despite the fact that songbirds are currently much less transgenically tractable than rodent models, controlled cellular and molecular studies relying on targeted genetic intervention are available in the zebra finch, and protocols for long-term transgene expression are currently being developed for songbirds [55]. As a first validation of these efforts, Haesler et al. [56] showed that lentivirally-mediated knockdown of FOXP2 in area X of developing zebra finches prior to song learning results in incomplete and inaccurate imitation of tutor song. The authors demonstrated that virus-mediated RNA interference can be reliably used to alter gene levels in songbird circuitry at a critical time point during song learning.

Future functional in vivo approaches of genetic intervention in song circuitry are expected to similarly evaluate the cellular and behavioral consequences of targeted delivery of over- and underexpressing CNTNAP2 genetic constructs during the formation of neural circuitry for song. As uncovered by the gene expression studies presented above, the targets for these genetic manipulations will include key nuclei specialized for song learning and production (LMAN, RA, area X). 
Given that the cellular and synaptic changes that occur in these specialized brain areas during song learning are well-understood, targeted genetic intervention in these brain subregions should illuminate the role of $C N T$ $N A P 2$ in key aspects of vocal development. Finally, it will be equally important to determine whether similar functional genetic links exist between FOXP2 and CNTNAP2 in songbird, as it has been shown in human, with FOXP2 acting to dramatically repress CNTNAP2 levels in human neuronal-like cell lines [31]. In particular, these latter functional analyses have the potential to ultimately uncover any human unique or shared neuromolecular pathways for speech and birdsong.

\section{Conclusion}

In summary, the songbird model offers an important advantage over traditional laboratory animals, in that it enables the study of vocal learning, a key subcomponent of human language. Given its experimental tractability, the zebra finch songbird species is fully amenable to controlled molecular and cellular studies that are not ethical or practical in humans and that are expected to illuminate the biological pathways underscoring learned vocal communication. As a first test, the study of FOXP2 and its transcriptional target in human, CNTNAP2, in the songbird model, revealed that the two language-related molecules are expressed at key locations and time points during the stages of song learning, suggesting a direct involvement in the sensorimotor processes underlying song learning and production. These findings now provide impetus for developing targeted functional investigations to probe the roles of these candidate molecules in the zebra finch neural circuitry for song. Moreover, the possibility that FOXP2 and CNTNAP2 may be functionally linked in songbird, as it has been shown in human, may ultimately reveal any unique or shared molecular and cellular pathways for speech and birdsong. Finally, controlled in vivo functional analyses probing the role of autism-susceptibility and language-related genes in the songbird circuitry for learned vocalization are expected to reveal basic molecular and cellular mechanisms at play in disorders of human speech and language such as ASD.

\section{References}

[1] S.E. Fisher, Tangled webs: Tracing the connections between genes and cognition, Cognition 101 (2006), 270-297.
[2] D.F. Newbury and A.P. Monaco, Genetic advances in the study of speech and language disorders, Neuron 68 (2010), 309-320.

[3] E. Fombonne, Epidemiology of pervasive developmental disorders, Pediatr Res 65 (2009), 591-598.

[4] B.S. Abrahams and D.H. Geschwind, Advances in autism genetics: On the threshold of a new neurobiology, Nat Rev Genet 9 (2008), 341-355.

[5] M.W. State and P. Levitt, The conundrums of understanding genetic risks for autism spectrum disorders, Nat Neurosci 14 (2011), 1499-1506.

[6] J. Peça, C. Feliciano, J.T. Ting, W. Wang, M. F. Wells, T.N. Venkatraman, C.D. Lascola, Z. Fu and G. Feng, Shank3 mutant mice display autistic-like behaviours and striatal dysfunction, Nature 472 (2011), 437-442.

[7] S. Jamain, K. Radyushkin, K. Hammerschmidt, S. Granon, S. Boretius, F. Varoqueaux, N. Ramanantsoa, J. Gallego, A. Ronnenberg, D. Winter, J. Frahm, J. Fischer, T. Bourgeron, H. Ehrenreich and N. Brose, Reduced social interaction and ultrasonic communication in a mouse model of monogenic heritable autism, Proc Natl Acad Sci U S A 105 (2008), 17101715.

[8] P.H. Patterson, Modeling autistic features in animals, Pediatr Res 69 (2011), 34R-40R.

[9] F. Nottebohm and A.P. Arnold, Sexual dimorphism in vocal control areas of the songbird brain, Science 194 (1976), 211213.

[10] A.P. Arnold, F. Nottebohm and D.W. Pfaff, Hormone concentrating cells in vocal control and other areas of the brain of the zebra finch (Poephila guttata), J Comp Neurol 165 (1976), 487-511.

[11] M.S. Brainard and A.J. Doupe, What songbirds teach us about learning, Nature 417 (2002), 351-358.

[12] A.J. Doupe and P.K. Kuhl, Birdsong and human speech: Common themes and mechanisms, Annu Rev Neurosci 22 (1999), 567-631.

[13] P.K. Kuhl, Human speech and birdsong: Communication and the social brain, Proc Natl Acad Sci U S A 100 (2003), 96459646.

[14] R.S. Waldstein, Effects of postlingual deafness on speech production: implications for the role of auditory feedback, $J$ Acoust Soc Am 88 (1990), 2099-2114.

[15] F. Nottebohm, T.M. Stokes and C.M. Leonard, Central control of song in the canary, Serinus canaries, J Comp Neurol 165 (1976), 457-486.

[16] J.M. Wild, Neural pathways for the control of birdsong production, J Neurobiol 33 (1997), 653-670.

[17] S.W. Bottjer, E.A. Miesner and A.P. Arnold, Forebrain lesions disrupt development but not maintenance of song in passerine birds, Science 224 (1984), 901-903.

[18] F. Sohrabji, E.J. Noredeen and K.W. Nordeen, Selective impairment of song learning following lesions of a forebrain nucleus in the juvenile zebra finch, Behav Neural Biol 53 (1990), 51-63.

[19] C. Scharff and F. Nottebohm F. 1991, A comparative study of the behavioral deficits following lesions of various parts of the zebra finch song system: implications for vocal learning, J Neurosci 11 (1991), 2896-2913.

[20] G.E. Vates and F. Nottebohm, Feedback circuitry within a song-learning pathway, Proc Natl Acad Sci U S A 92 (1995), 5139-5143.

[21] E.S. Fortune, D. Margoliash, Parallel Pathways and Convergence Onto HVc and Adjacent Neostriatum of Adult Zebra Finches (Taeniopygia guttata), J Comp Neurol 360 (1995), 413-441. 
[22] E.F. Foster and S.W. Bottjer, Axonal connections of the high vocal center and surrounding cortical regions in juvenile and adult male zebra finches, J Comp Neurol 397 (1998), 118-138.

[23] J.W. Lewis, S.M. Ryan, A.P. Arnold and L.L. Butcher, Evidence for a catecholaminergic projection to area $\mathrm{X}$ in the zebra finch, J Comp Neurol 196 (1981), 347-354.

[24] M.A. Farries, Organization and electrophysiological properties of an avian basal ganglia structure essential for song learning, Ph.D. Dissertation, University of Pennsylvania, 2002.

[25] A.M. Graybiel, T. Aosaki, A.W. Flaherty and M. Kimura, The basal ganglia and adaptive motor control, Science 265 (1994), 1826-1831.

[26] W. Schultz, Predictive reward signal of dopamine neurons, $J$ Neurophysiol 80 (1998), 1-27.

[27] C.S. Lai, S.E. Fisher, J.A. Hurst, F. Vargha-Khadem and A.P. Monaco, A forkhead-domain gene is mutated in a severe speech and language disorder, Nature 413 (2001), 519-523.

[28] I. Teramitsu, L.C. Kudo, S.E. London, D.H. Geschwind and S.A. White, Parallel FoxP1 and FoxP2 expression in songbird and human brain predicts functional interaction, J Neurosci $\mathbf{2 4}$ (2004), 3152-3163

[29] E. Spiteri, G. Konopka, G. Coppola, J. Bomar, M. Oldham, J. Ou, S.C. Vernes, S.E. Fisher, B. Ren and D.H. Geschwind, Identification of the transcriptional targets of FOXP2, a gene linked to speech and language, in developing human brain, $A m$ J Hum Genet 81 (2007), 1144-1157.

[30] S.C. Vernes, E. Spiteri, J. Nicod, M. Groszer, J.M. Taylor, K.E. Davies, D.H. Geschwind and S.E. Fisher, High-throughput analysis of promoter occupancy reveals direct neural targets of FOXP2, a gene mutated in speech and language disorders, Am J Hum Genet 81 (2007), 1232-1250.

[31] S.C. Vernes, D.F. Newbury, B.S. Abrahams, L. Winchester, J. Nicod, M. Groszer, M. Alarcón, P.L. Oliver, K.E Davies, D.H. Geschwind, A.P. Monaco and S.E. Fisher, A functional genetic link between distinct developmental language disorders, $N$ Engl J Med 359 (2008), 2337-2345.

[32] M. Alarcón, B.S. Abrahams, J.L. Stone, J.A. Duvall, J.V. Perederiy, J.M. Bomar, J. Sebat, M. Wigler, C.L. Martin, D.H. Ledbetter, S.F. Nelson, R.M. Cantor and D.H. Geschwind, Linkage, association, and gene-expression analyses identify CNTNAP2 as an autism-susceptibility gene, Am J Hum Genetics 82 (2008), 150-159.

[33] D.E. Arking, D.J. Cutler, C.W. Brune, T.M. Teslovich, K. West, M. Ikeda, A. Rea, M. Guy, S. Lin, E.H. Cook and A. Chakravarti, A common genetic variant in the neurexin superfamily member CNTNAP2 increases familial risk of autism, Am J Hum Genet 82 (2008), 160-164.

[34] B. Bakkaloglu, B.J. O'Roak, A. Louvi, A.R. Gupta, J.F. Abelson, T.M. Morgan, K. Chawarska, A. Klin, A.G ErcanSencicek, A.A. Stillman, G. Tanriover, B.S. Abrahams, J.A. Duvall, E.M. Robbins, D.H. Geschwind, T. Biederer, M. Gunel, R.P. Lifton and M.W. State, Molecular cytogenetic analysis and resequencing of contactin associated protein-like 2 in autism spectrum disorders, Am J Hum Genet 82 (2008), 165-173.

[35] M. Alarcón, R.M. Cantor, J. Liu, T.C. Gilliam, the Autism Genetic Research Exchange Consortium and D.H. Geschwind, Evidence for a language quantitative trait locus on chromosome $7 \mathrm{q}$ in multiplex autism families, Am J Hum Genet $\mathbf{7 0}$ (2002), 60-71.

[36] K.A. Strauss, E.G. Puffenberg, M.J. Huentelman, S. Gottlieb, S.E. Dobrin, J.M. Parod, D.A. Stephan and D.H. Morton, Recessive symptomatic focal epilepsy and mutant contactin- associated protein-like 2, N Engl J Med 354 (2006), 13701377.

[37] J.I. Friedman, T. Vrijenhoek, S. Markx, I.M. Janssen, W.A. van der Vliet, B.H.W. Faas, N.V. Knoers, W. Cahn, R.S. Kahn, L. Edelmann, K.L. Davis, J.M. Silverman, H.G. Brunner, A.G. van Kessel, C. Wijmenga, R.A. Ophoff and J.A. Veltman, CNTNAP2 gene dosage variation is associated with schizophrenia and epilepsy, Mol Psychiatry 13 (2007), 261266.

[38] S. Poliak, L. Gollan, R. Martinez, A. Custer, S. Einheber, J.L. Salzer, J.S. Trimmer, P. Shrager and E. Peles, Caspr2, a new member of the neurexin superfamily, is localized at the juxtaparanodes of myelinated axons and associates with $\mathrm{K}+$ channels, Neuron 24 (1999), 1037-1047.

[39] S. Poliak, L. Gollan, D. Salomon, E.O. Berglund, R. Ohara, B. Ranscht and E. Peles, Localization of Caspr 2 in myelinated nerves depends on axon-glia interactions and the generation of barriers along the axon, J Neurosci 21 (2001), 7568-7575.

[40] S. Poliak, D. Salomon, H. Elhanany, H. Sabanay, B. Kiernan, L. Pevny, C.L. Stewart, X. Xu, S.Y. Chiu, P. Shrager, A.J. Furley and E. Peles, Juxtaparanodal clustering of Shaker-like $\mathrm{K}+$ channels in myelinated axons depends on Caspr2 and TAG1, J Cell Biol 162 (2003), 1149-1160.

[41] B.S. Abrahams, D. Tentler, J.V. Perederiy, M.C. Oldham, G. Coppola and D.H. Geschwind, Genome-wide analyses of human perisylvian cerebral cortical patterning, Proc Natl Acad Sci U S A 104 (2007), 17849-17854.

[42] O. Peñagarikano, B.S. Abrahams, E.I. Herman, K.D. Winden, A. Gdalyahu, H. Dong, L.I. Sonnenblick, R. Gruver, J. Almajano, A. Bragin, P. Golshani, J.T. Trachtenberg, E. Peles and D.H. Geschwind, Absence of CNTNAP2 Leads to Epilepsy, Neuronal Migration Abnormalities, and Core Autism-Related Deficits, Cell 147 (2011), 235-246.

[43] S.C. Panaitof, B.S. Abrahams, H. Dong, D.H. Geschwind and S.A. White, Language-related Cntnap2 gene is differentially expressed in sexually dimorphic song nuclei essential for vocal learning in songbirds, J Comp Neurol 518 (2010), 1995-2018.

[44] M.A. Farries and D.J. Perkel, Electrophysiological properties of avian basal ganglia neurons recorded in vitro, J Neurophysiol 84 (2000), 2502-2513.

[45] M.A. Farries and D.J. Perkel, A telencephalic nucleus essential for song learning contains neurons with physiological characteristics of both striatum and globus pallidus, J Neurosci 22 (2002), 3776-3787.

[46] M. Konishi and E. Akutagawa, Neuronal growth, atrophy and death in a sexually dimorphic song nucleus in the zebra finch brain, Nature 315 (1985), 145-147.

[47] R. Mooney and M. Rao, Waiting periods versus early innervation: the development of axonal connections in the zebra finch song system, J Neurosci 14 (1994), 6532-6543.

[48] M.C. Condro, J.E. Miller and S.A. White, Autism susceptibility gene contactin associated protein-like 2 expression in a songbird model for vocal learning, Society for Neuroscience Abstracts, 2011.

[49] C. Winograd, D. Clayton and S. Ceman, Expression of fragile $\mathrm{X}$ mental retardation protein within the vocal control system of developing and adult male zebra finches, Neuroscience $\mathbf{1 5 7}$ (2008), 132-142.

[50] W.T. O'Donnell and S.T. Warren, A decade of molecular studies of fragile X syndrome, Annu Rev Neurosci 25 (2002), 315338.

[51] L. Abbeduto, N. Brady and S.T. Kover, Language development and fragile $\mathrm{X}$ syndrome: profiles, syndrome specificity, and 
within-syndrome differences, Ment Retard Dev Disabil Res Rev 13 (2007), 36-46.

[52] L.H. Finestack, E.K. Richmond and L. Abbeduto, Language development in individuals with Fragile $\mathrm{X}$ syndrome, Top Lang Disord 29 (2009), 133-148.

[53] P. Lewis, L Abbeduto, M. Murphy, E. Richmond, N. Giles, L. Bruno and S. Schroeder, Cognitive, language and socialcognitive skills of individuals with fragile $\mathrm{X}$ syndrome with and without autism, J Intellect Disabil Res 50 (2006), 532-545.

[54] I. Teramitsu and S.A. White, FoxP2 regulation during undi- rected singing in adult songbirds, J Neurosci 26 (2006), 73907394.

[55] R. J. Agate, B. B. Scott, B. Haripal, C. Lois and F. Nottebohm, Transgenic songbirds offer an opportunity to develop a genetic model for vocal learning, Proc Natl Acad Sci U S A 106 (2009), 17963-17967.

[56] S. Haesler, C. Rochefort, B. Georgi, P. Licznerski, P. Osten and C. Scharff, Incomplete and inaccurate vocal imitation after knockdown of FoxP2 in songbird basal ganglia nucleus Area X, PLoS Biol 5 (2007), e321. 


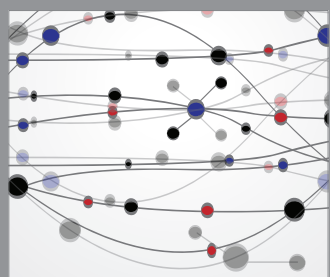

The Scientific World Journal
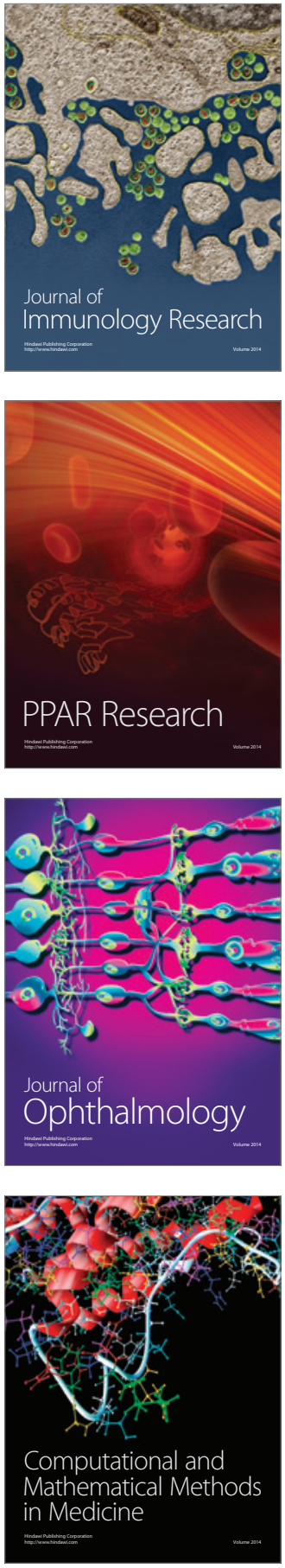

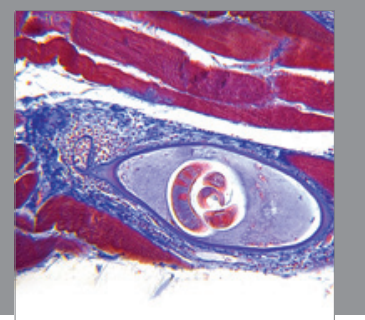

Gastroenterology

Research and Practice
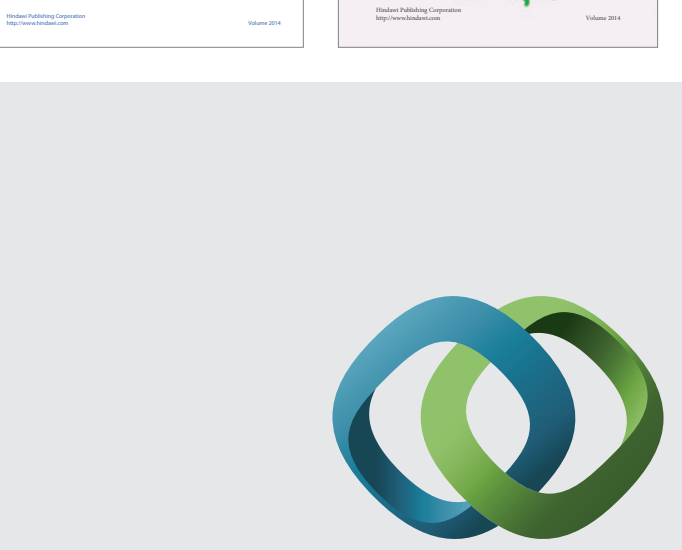

\section{Hindawi}

Submit your manuscripts at

http://www.hindawi.com
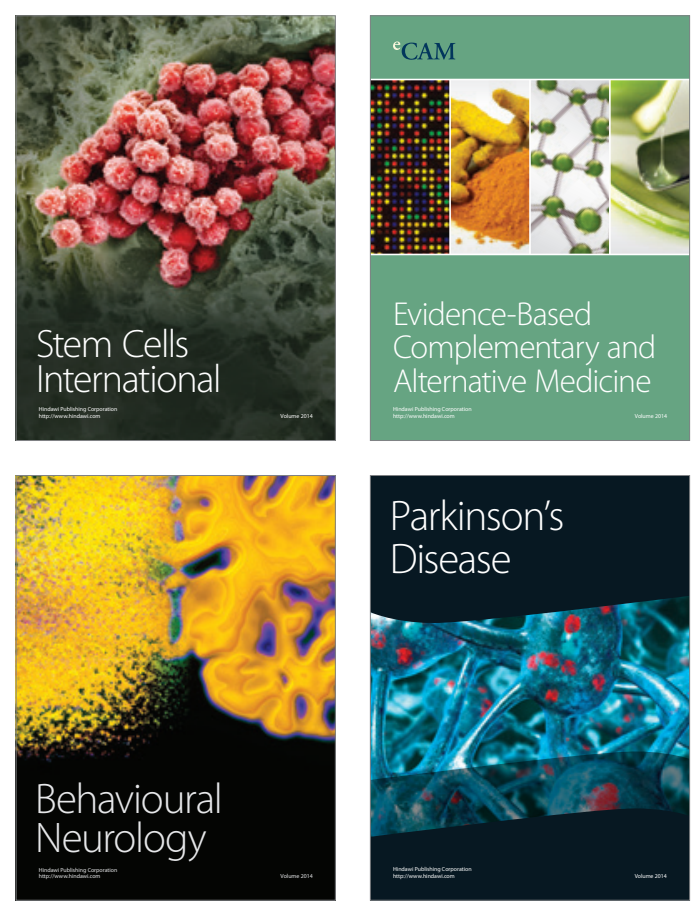

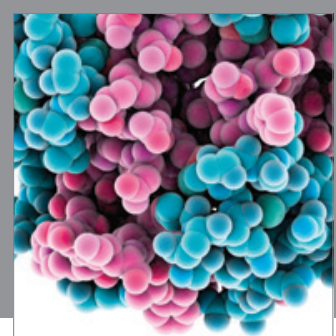

Journal of
Diabetes Research

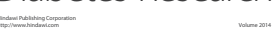

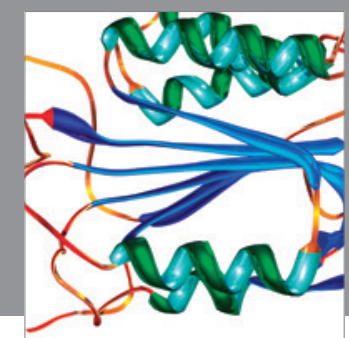

Disease Markers
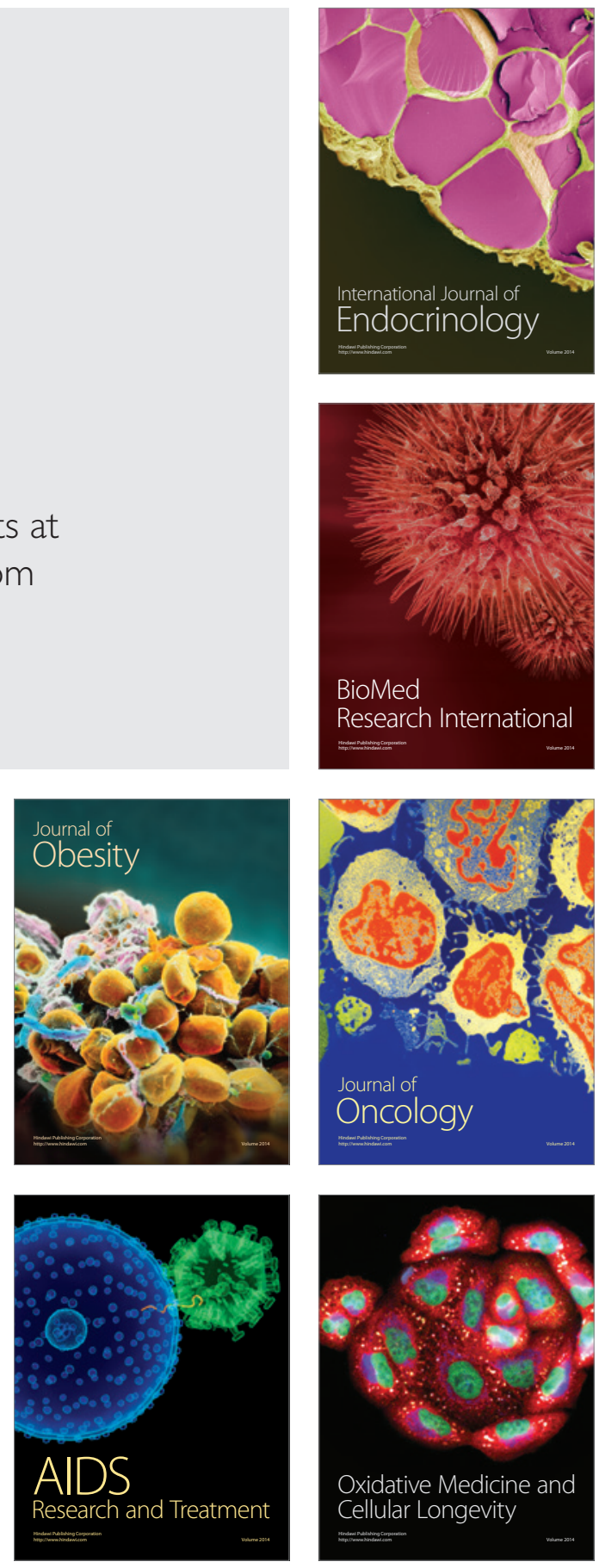